\title{
Opportunities for big data in conservation and sustainability
}

\author{
Rebecca K. Runting ${ }^{1 凶}$, Stuart Phinn (10 ${ }^{2}$, Zunyi Xie ${ }^{2}$, Oscar Venter ${ }^{3} \&$ \\ James E. M. Watson (1D) 2,4
}

Big data reveals new, stark pictures of the state of our environments. It also reveals 'bright spots' amongst the broad pattern of decline and-crucially-the key conditions for these cases. Big data analyses could benefit the planet if tightly coupled with ongoing sustainability efforts.

This big data revolution, which encompasses techniques to capture, process, analyse and visualize large datasets in a rapid timeframe, has led to an explosion in data variety over the last five decades (Fig. 1a). Significant advances in data growth in the bio-geophysical sciences have allowed scientists to discover, analyse and understand environmental changes at micro to global scales, and separate out what is human-driven. As a consequence, the state and trends of the environment is increasingly becoming a focus of big data applications (Fig. 1b). Here, we discuss the trends emerging from these environmental analyses (including the derived data products) and propose a way forward to harness these technologies to mitigate global environmental declines.

\section{Environmental changes revealed by big data}

Almost invariably, the advances in big data analyses confirm planetary declines and, in most cases, reveal that declines are worse than previously indicated (Fig. 1b). For example, a landmark collaboration between NASA and the European Space Agency integrated the Antarctic ice sheet's changing volume, flow and gravitational attraction to model its surface mass balance, which revealed Antarctica lost $2720 \pm 1390 \mathrm{Mg}$ of ice between 1992 and 2017 (equating to a sea-level rise of $7.6 \pm 3.9 \mathrm{~mm})^{2}$. Yet only a few years prior, the IPCC stated that we have medium confidence in model projections of a future Antarctic SMB [surface mass balance] increase, implying a negative contribution to GMSL [global mean sea level] rise' (p. $\left.1155^{10}\right)$. Similarly, efforts to create a Red List of Ecosystems - an emerging methodology using multiple lines of evidence to assess the threat status of ecosystems ${ }^{11}$ - has already revealed some alarming results. For instance, in the Americas and the Caribbean, $85 \%$ of the forest area and $80 \%$ of forest types are potentially threatened ${ }^{12}$. While these methodical developments are impressive and urgently needed, they reveal a stark picture for the environment.

However, analyses of big data have also revealed 'bright spots' amongst the broad pattern of decline and-crucially-identify the key drivers, including deliberate policy interventions. For instance, while Hansen et al. ${ }^{5}$ revealed dramatic declines in forest extent across the globe, forest loss in Brazil was decreasing by $1318 \mathrm{~km}^{2} \mathrm{y}^{-1}$ through the 12 year period to 2012 , primarily due to a progressive legal framework covering forests during the study period (although the change

\footnotetext{
${ }^{1}$ School of Geography, The University of Melbourne, Parkville, VIC, Australia. ${ }^{2}$ School of Earth and Environmental Sciences, The University of Queensland Brisbane, QLD, Australia. ${ }^{3}$ Natural Resource and Environmental Studies Institute, University of Northern British Columbia, Prince George, BC, Canada.

${ }^{4}$ Wildlife Conservation Society, Global Conservation Program, New York, NY, USA. ${ }^{凶}$ email: rebecca.runting@unimelb.edu.au
} 
a Timeline of technological change

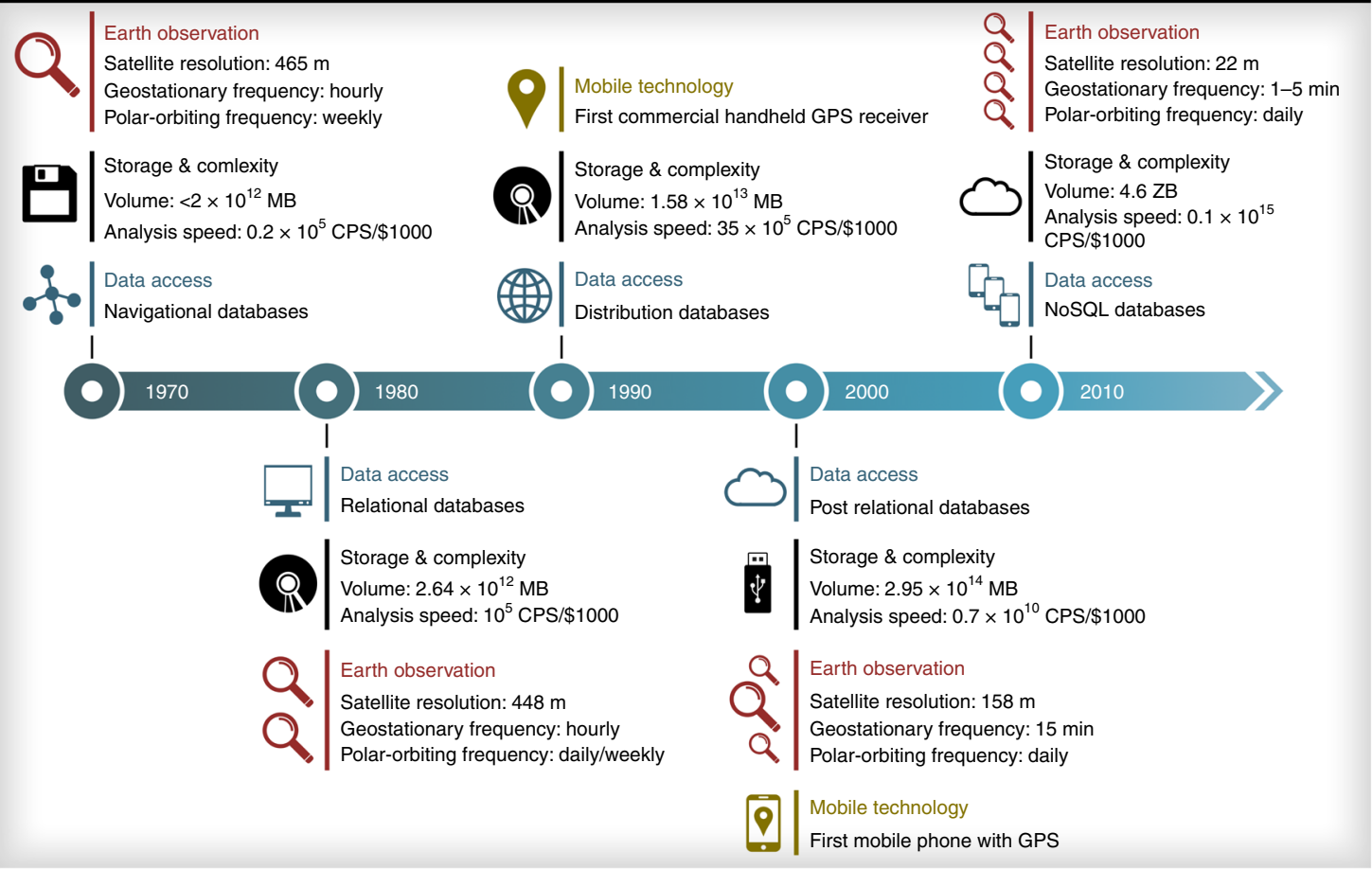

b Timeline of environmental change

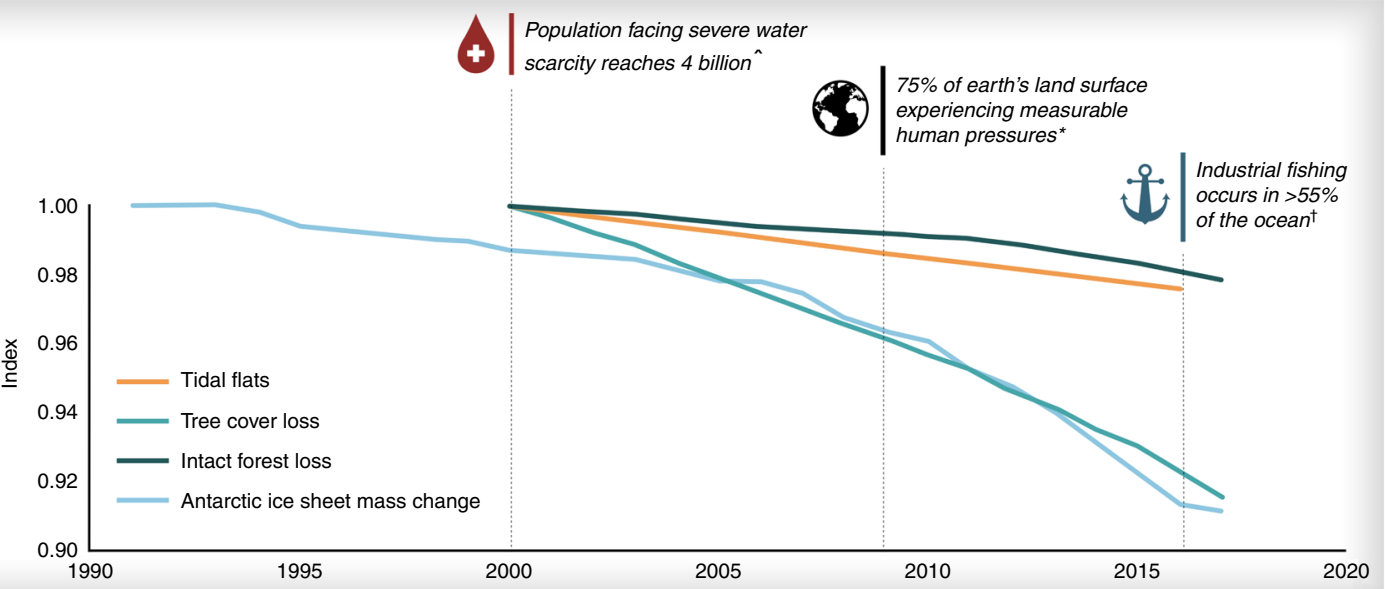

Fig. 1 Timeline of selected technological and environmental changes. (a) The volume of data produced has grown exponentially and is expected to soon reach 40 Zettabytes (40 trillion Gigabytes). Such data generation is only possible due to the concurrent growth in data storage and computing speed, which has moved from the floppy disk ( 1 calculations per second per $\$ 1000)$ to cloud-based storage ( $>10^{15}$ calculations) in last 30 years ${ }^{1}$. Despite this exponential growth in technological capacity, and increasing environmental applications, our planet is still facing serious environmental declines (b). All environmental declines shown are sourced from prior studies (as detailed below) and are indexed relative to their state in the first year plotted (i.e., dividing by the first value in each time series), with the exception of Antarctic ice sheet mass change ${ }^{2}$, which was indexed against expected (BAU) loss by $2100\left(81 \mathrm{~cm}\right.$ sea-level rise equivalent $\left.{ }^{3}\right)$. Tidal flats represent the overall decline across the globe for time period, and does not show annual fluctuations ${ }^{4}$. Intact Forest Landscapes and tree cover loss does not take into account gains ${ }^{5,6}$. Note the index on the $y$-axis is only shown for the range 0.9-1. ${ }^{\circ}$ Data from Global fishing watch ${ }^{7}$. $B a s e d$ on the global human footprint ${ }^{8}$. ${ }^{\wedge}$ For at least 1 month a year over the period $1996-2005^{9}$.

in government in 2019 has since reversed this trend). Similarly, recent analyses of satellite data by Chen et al. ${ }^{13}$ showed that direct human land-management has led to greening over large expanses in China and India. Much of the gains in China were from forest (rather than agriculture), which was driven by ambitious national policies for afforestation and forest conservation underpinned by payments for these ecosystem services ${ }^{14}$. Biophysical drivers can also produce positive trends. For instance, an analysis of derived big data products revealed that increased precipitation in the
Tibetan Plateau over the last four decades has resulted in vegetation greening ${ }^{15}$. While this greening is good for carbon sequestration, it is important to note that it may ultimately disrupt the existing ecosystem and species. Overall, the mean human pressure in the world's 24 most developed countries decreased from 1993-2009, potentially due to rural-to-urban migration and restoration programs ${ }^{8}$. Despite these regional improvements, they are not yet sufficient to reverse the global trend towards environmental decline. 


\section{Coupling big data and the global sustainability agenda}

Analyses of big data are clearly essential for highlighting declines in Earth's environment and its capacity to support humans. Yet, these impressive advances will not benefit the planet and its people unless we can act to achieve sustainability goals, so it is essential that big data coalesce with ongoing efforts to achieve sustainability. Unfortunately, this is not yet the case for many national and international policy processes. Despite international agreement on the Aichi Biodiversity Targets (which form the strategic plan for the Convention for Biological Diversity), many nations are not taking advantage of the availability of big data and derived products to achieve these goals. Numerous spatial data layers necessary for implementing the Aichi Targets already exist at the national or global scale, yet $80 \%$ of the 5 th round of National Reports on these targets contained no actionable maps ${ }^{16}$. In most cases, a map of environmental phenomena requires additional analysis and combination with other datasets before it can be used to directly inform decisions, which may require additional resources (e.g., technical, financial) that are challenging for some less-developed countries to acquire.

It is essential that barriers to analysing big data and accessing derived products are removed. This could take the form of accessible outputs, such as the Global Fishing Watch, Global Forest Watch and global inter-tidal websites (www.globalfishingwatch. org, www.globalforestwatch.org, www.intertidal.app), or userfriendly analysis tools, such as REMAP - a free application that utilises the storage and analysis capacity of Google Earth Engine to map land cover change (www.remap-app.org). While there is a growing trend towards open data, some authorities still maintain barriers to access including fees, substantial delays, or incomplete data releases. For example, in the Australian state of Queensland, which has one of the most dramatic recent land clearing legacies, governmental reporting on vegetation clearance is often delayed by months despite the fact it could be monitored monthly. Ensuring the timely availability of big data products will require a sincere commitment to a shared vision for open data, before more procedural issues such as data management standards can be addressed. A number of international organisations are addressing this, with the WMO (World Meteorological Office, https://public. wmo.int/), possibly one of the longest running and best examples of what is possible from real global collaborations across many countries, while the GEO (Group on Earth Observations, www. earthobservations.org) links 108 nation's earth observation capabilities to address sustainable development goals. Further, as international accords for conservation and sustainability are operationalised by countries, it is vital to engage national- and international-level decision-makers in government and industry in the generation of global datasets to ensure they are able to be used by all to make effective decisions.

The private sector is increasingly making influential environmental decisions and some large companies are committing to sustainability in their supply chains. Examples include 'zerodeforestation' and sustainably sourced palm oil pledges from Nestlé and McDonalds. Tracking the full supply chain for large corporations requires the use of big data analytics, particularly to balance the multiple objectives corporations seek from their supply chains (such as reducing carbon emissions and increasing profitability ${ }^{17}$. The use of geospatial, earth observation and many other data will be essential for transparency and monitoring compliance by certification bodies, environmental NGOs and the corporations themselves. Ongoing research, particularly integrating qualitative data, is likely to enable an even closer coupling of big data analytics and sustainable supply chain management. Ultimately, the timely use of big data will be critical to placing commodity production and trade on a sustainable pathway.
Big data now must be harnessed for ecological forecasting to improve decision-making, in both the public and private sectors. Monitoring environmental change in near real-time can be beneficial if there is capacity for action at a similar temporal scale, which is often not the case. However, useful applications are emerging. For instance, Chen et al. ${ }^{18}$ investigated links between sea surface temperatures and interannual changes in fire activity in South America and forecasted the regional severity of the fire season with a 3-5 month lead time. Across the Middle East, Levin and colleagues ${ }^{19}$ integrated temporal data on night lights, wildfire, news databases and Flickr photos to identify World Heritage Sites affected by conflict in near real-time. In the marine realm, an automated vessel tracking and monitoring system (which uses a constellation of satellites and terrestrial receivers) can be used to inform models, which predict illegal fishing activity in realtime $^{20}$. Identifying patterns of suspicious behaviour has allowed governments to conduct targeted investigations of particular vessels that may be undertaking illegal activity in their waters. Such 'early warning systems', should be given a platform in relevant management agencies-and closely linked with management action-to harness vast potential of these methods to improve outcomes for nature and people.

To ensure these close links with environmental decisionmaking, the acquisition and analysis of big data must be solution focused and address sustainability challenges while engaging with decision-makers and those affected by such decisions. From documenting our planet's greenness to detecting where resources are being illegally harvested, big data analyses can now place detailed evidence of rapid environmental change in the hands of entities capable of management action. Ultimately, there must be a tight coupling of big data analyses and the sustainability agenda to ensure we do not run out of time and space to save our environment-and ourselves.

Received: 6 February 2020; Accepted: 1 April 2020; Published online: 24 April 2020

\section{References}

1. Hilbert, M. \& López, P. The world's technological capacity to store, communicate, and compute information. Science 332, 60-65 (2011).

2. Shepherd, A. et al. Mass balance of the Antarctic Ice Sheet from 1992 to 2017. Nature 558, 219-222 (2018).

3. Bindschadler, R. A. et al. Ice-sheet model sensitivities to environmental forcing and their use in projecting future sea level (the SeaRISE project). J. Glaciol. 59, 195-224 (2013).

4. Murray, N. J. et al. The global distribution and trajectory of tidal flats. Nature 565, 222-225 (2019).

5. Hansen, M. C. et al. High-resolution global maps of 21st-century forest cover change. Science 342, 850-853 (2013).

6. Potapov, P. et al. The last frontiers of wilderness: tracking loss of intact forest landscapes from 2000 to 2013. Sci. Adv. 3, e1600821 (2017).

7. Kroodsma, D. A. et al. Tracking the global footprint of fisheries. Science 359, 904-908 (2018).

8. Venter, O. et al. Sixteen years of change in the global terrestrial human footprint and implications for biodiversity conservation. Nat. Commun. 7, 12558 (2016).

9. Mekonnen, M. M. \& Hoekstra, A. Y. Four billion people facing severe water scarcity. Sci. Adv. 2, e1500323 (2016).

10. Church, J. A. et al. Sea level change. in Climate Change 2013: The Physical Science Basis. Contribution of Working Group I to the Fifth Assessment Report of the Intergovernmental Panel on Climate Change (eds Stocker, T. F. et al.) (Cambridge University Press, Cambridge, 2013).

11. Bland, L. M. et al. Using multiple lines of evidence to assess the risk of ecosystem collapse. Proc. Biol. Sci. 284, 20170660 (2017).

12. Ferrer-Paris, J. R. et al. An ecosystem risk assessment of temperate and tropical forests of the Americas with an outlook on future conservation strategies. Conserv. Lett. https://doi.org/10.1111/conl.12623 (2019)

13. Chen, C. et al. China and India lead in greening of the world through land-use management. Nat. Sustain 2, 122-129 (2019). 
14. Bryan, B. A. et al. China's response to a national land-system sustainability emergency. Nature 559, 193-204 (2018).

15. Zhang, W., Zhou, T. \& Zhang, L. Wetting and greening Tibetan Plateau in early summer in recent decades. J. Geophys. Res. Atmos. 122, 5808-5822 (2017).

16. Ervin, J. et al. Are We Counting on Nature? An Analysis of Spatial Data included in Post-2010 National Biodiversity Strategies and Action Plans and 5th National Reports. (UNDP, 2017).

17. Zhao, R., Liu, Y., Zhang, N. \& Huang, T. An optimization model for green supply chain management by using a big data analytic approach. J. Clean. Prod. 142, 1085-1097 (2017).

18. Chen, Y. et al. Forecasting fire season severity in South America using sea surface temperature anomalies. Science 334, 787-791 (2011).

19. Levin, N., Ali, S., Crandall, D. \& Kark, S. World Heritage in danger: big data and remote sensing can help protect sites in conflict zones. Glob. Environ. Chang. 55, 97-104 (2019).

20. Ford, J. H. et al. Detecting suspicious activities at sea based on anomalies in automatic identification systems transmissions. PLoS ONE 13, e0201640 (2018).

\section{Acknowledgements}

This research was supported by Australian Research Council Discovery Project grant no. DP160101397. We would like to thank B. Alexander Simmons for work on Fig. 1, and Peter Scarth for useful discussions and feedback.

\section{Author contributions}

J.E.M.W. and S.P. conceptualised the idea for the manuscript, which was refined by R.K.R., Z.X. and O.V.; R.K.R. led the development of the figure and writing of the manuscript with input from J.E.M.W., S.P., Z.X. and O.V.

\section{Competing interests}

The authors declare no competing interests.

\section{Additional information}

Correspondence and requests for materials should be addressed to R.K.R.

Reprints and permission information is available at http://www.nature.com/reprints

Publisher's note Springer Nature remains neutral with regard to jurisdictional claims in published maps and institutional affiliations.

(c) (i) Open Access This article is licensed under a Creative Commons Attribution 4.0 International License, which permits use, sharing, adaptation, distribution and reproduction in any medium or format, as long as you give appropriate credit to the original author(s) and the source, provide a link to the Creative Commons license, and indicate if changes were made. The images or other third party material in this article are included in the article's Creative Commons license, unless indicated otherwise in a credit line to the material. If material is not included in the article's Creative Commons license and your intended use is not permitted by statutory regulation or exceeds the permitted use, you will need to obtain permission directly from the copyright holder. To view a copy of this license, visit http://creativecommons.org/ licenses/by/4.0/.

(C) The Author(s) 2020 